\title{
Influence of Light Through Optical Glasses on Electroencephalogram
}

\author{
John William Carey Medithe*, Usha Rani Nelakuditi \\ Department of Electronics and Communications Engineering, Vignan's University, Guntur, Andhra Pradesh, INDIA.
}

\begin{abstract}
Background: In General, optical powered glasses are used to have good focus and clear vision. But, this practice may also bring noticeable variation in light intensity which in turn affects neural behavior. These changes can be interpreted from the EEG recordings. Thus, in this present study, the grounds for amendments in different physiological variables due to light intensity through optical glasses experienced by the subject have been reviewed and verified. Experiment: Changes in light intensity through optical powered glasses have been demonstrated using a Light Dependent Resistor (LDR) test for various cases. From this study, it was found that positive powered optical glass increases the light intensity falling on an eye, while negative powered optical glass reduces light intensity. Case Study: A case study was performed using experimental and subjective analysis were made to confirm that misfocused or unclear vision bring changes in frontal frequency and makes subject fatigue and eyes strain which is prone to severe headache and development of seizures. Recommendations: It is to be noted by the EEG practitioner to confirm that, whether optical powered glasses used by the subject is to be match with refractive error of an eye. If not, the frontal frequency gets increased and may suspect as seizures or other any abnormality.
\end{abstract}

Key words: Electroencephalogram, Light, Light Dependent Resistor, Optical glass, Neurophysiology

\section{INTRODUCTION}

Human brain is systematized in controlling and integrating various sensing organs in the human system. Brain consists of millions of neurons; these neurons are special group of cells that are capable of being excited and the action potential generated and induced over the scalp. Electroencephalogram is a significant medical imaging test for the study and interpretation of electrical activity of the brain with respect to time. These electric potentials would be an array of microvolt. Subdural electrodes are used for invasive studies during surgery. Visible light is a part of an electromagnetic spectrum sensed by the humans by their Ocular sensor. There are various changes which occur in human physiology when exposed to different intensities of light. There are noticeable and major alterations in subjects Ocular Physiology and Neurology due to light exposure. The changes in light intensity which illuminate on to the human eye also reflect on electroencephalographic readings. ${ }^{1-3}$

Optical powered glasses are used by people to correct refractive errors of an eye. These optical powered glasses help them to have clear vision and proper focus to perform any visual task. An anomalous refractive condition such as myopia, hypermetropia and astigmatism of an eye makes an image fail to focus properly upon the retina. Myopia or nearsightedness is a result of the eyeball being too long than usual leading to misfocus the target object; this misfocused condition can be corrected by wearing negative powered optical glass. On the contrary, Hypermetropia or farsightedness occurs when the eyeball becomes shorter, and can be corrected by wearing positive powered optical glasses. Presbyopia and Astigmatism are other kinds of refractive errors which occur in eye due to age and other factors. When light enters the eye through these corrective lenses it brings noticeable changes in the human physiology and neuronal behavior. In this study, when a subject is exposed to light, remarkable changes in the human physiological parameters like change in release of percentage of melatonin hormone, change in core body temperature and more were observed which in turn affects the neuronal
Submission Date: 18-11-2016; Revision Date: 03-01-2017; Accepted Date: 15-02-2017

DOI: 10.5530/ijper.51.2s.50 Correspondence: John William Carey Medithe,

Department of Electronics and Communications Engineering, Vignan's University, Guntur, Andhra Pradesh, INDIA

Phone: +91 8977183538

E-mail: careymedithe@ gmail.com*

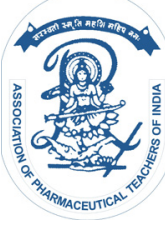

www.ijper.org 
measures such as change in EEG frequencies and EEG spectral density when exposed to light. To explore consequences due to light through optical glasses in neural behavior; alpha activity in occipital region is centrally weighted. This alpha activity is synchronized with intensity of light.

Previous literature describes the altering effects of light in various physiological parameters and EEG spectra when exposed to light with naked eye. ${ }^{4-6}$ But, in this study the variation of light through optical glasses are illustrated.

\section{Hypothesis of Variation in Light Intensity Through Optical Glasses}

Powered optical glass or spectacle lens is an optical device that alters the focus of a light beam through refraction. Optical lenses are different from normal lenses. The optical prescription given by the ophthalmologist is to correct the refractive errors of the human visual system. But, these corrective lenses used to overcome ametropic conditions cause a variation in light intensity experienced by the subjects. There are various components in the prescription suggested by the ophthalmologists such as spherical component which is used for focal correction and cylindrical component for fine tuning. Eyeglasses are convex in front and concave at the back surface. Positive powered lens is also identified as convex lens which is thicker in the middle and thinner at the edges. While negative powered lenses are concave lenses which are thicker on the edges than in the middle.

\section{Variations of light intensity through positive powered optical lenses}

Positive powered lens or converging lenses are suggested by the ophthalmologist to correct the refractive errors in the eye when subject experiences difficulty in focusing nearby objects. This difficulty occurs, when the light rays entering the eye focus behind the retina, rather than directly focusing on it. When the positive powered correcting lens is used, the light intensity coming from the light source fall on lens and gets converged at a point. It is observed that there is a variance in light experienced by the normal subject compared to subjects with positive powered lens, with same illumination conditions. The subjects who are having hypermetropia and got their refractive error corrected with positive powered lenses experienced more light intensity than the normal.

\section{Variations of light intensity through negative powered optical lenses}

Ophthalmologist suggests, Negative powered or diverging lens when the subject experiences the refractive error in the eye and faces difficulty in focusing the far objects. Here in this case, the light rays coming from the light source and falling on the diverging lens deviate from the principal axis. By wearing these types of glasses, there will be variation in light experienced by the subject with negative powered lens when compared to the normal subject. It is to be observed that subjects with myopia corrected with negative powered optical glass experiences the less light intensity with that of normal subjects in same illumination conditions.

\section{Light Dependent Resistor Test to Observe the Changes in Light Intensity Due to Optical Powered Glasses}

In order to check the above hypothesis, the variations in light intensity from a source like White LED or ambient light are processed through optical lenses of different powers. Here, to prove and verify that the wearable optical glasses alter the light intensity which enters into an eye. To examine the alterations in the intensity, Light dependent resistance test has been performed.

\section{Experimental Procedure}

A quality light dependent resistor was placed on the breadboard and it is connected to the multimeter. Initially, the illumination conditions of the LDR were calibrated to random value. The lenses of different prescriptions are obtained from an optical store are need to be placed at a uniform distance in front of the Light Dependent Resistor. Secondly, the lenses also kept at 3 $\mathrm{cm}, 5 \mathrm{~cm}$, and $7 \mathrm{~cm}$ distances in front of the Light Dependent Resistor. The change in reading for different powers of lenses placed in front of LDR is obtained in multimeter connected. This arrangement is shown in below Figure 1.

\section{RESULTS}

A resistance of $5.2 \mathrm{~K} \Omega$ is observed, when the LDR is placed in ambient light normal reading room illumination conditions when no lens or obstacle is kept before it, this observed resistance value is considered as a calibrated resistance. The alterations in readings when the powered lenses kept before the LDR are stated in Table 1.

\section{DISCUSSION}

The general exploit of the light dependent resistor (LDR) is to vary the resistance with respect to the light intensity falling on the face of the LDR. The resistance of the LDR reaches to its optimum, when the light intensity is not falling or poor luminance condition. The increment in the intensity of the light falling on the LDR leads to the decrement in resistance developed by the LDR. When the eyeglasses are placed before the face of the LDR, there is a noticeable resistance change observed in the multimeter. When lenses are placed before LDR, It is observed that the positive $(+)$ powered optical glass increases the light intensity falling on the LDR which results in reduction of

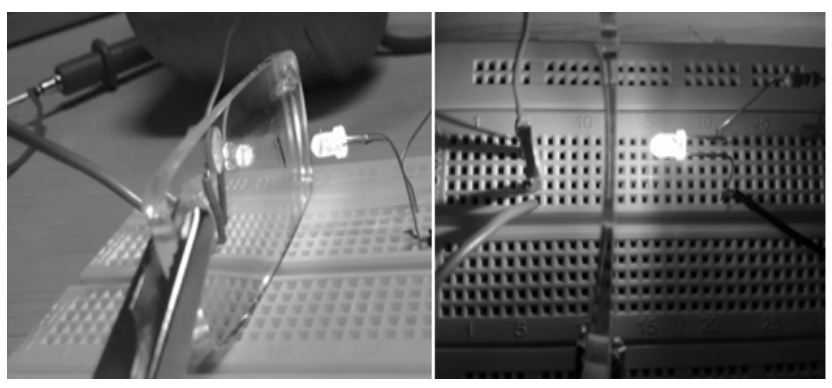

Figure 1: An LDR test to study the variation of light intensity through optical lenses 


\begin{tabular}{|c|c|}
\hline Prescription of lens (DIOPTERS) & LDR reading $(\mathrm{K} \Omega)$ \\
\hline+15 & 2.80 \\
\hline+12 & 3.15 \\
\hline Reading glasses Add +2 & 5.10 \\
\hline-1.75 Cyl & 5.64 \\
\hline$-2.5-0.5-X 90$ & 5.87 \\
\hline$-2.5-0.5-X 180$ & 5.80 \\
\hline$-3 \mathrm{Sph}$ & 5.97 \\
\hline$-3.5 \mathrm{Sph}$ & 6.12 \\
\hline
\end{tabular}

Table 2: LDR Reading for the different optical powered lens when LDR calibrated to $11.10 \mathrm{~K} \Omega$ and lens are kept at distances $3 \mathrm{~cm}, 5 \mathrm{~cm}, 7 \mathrm{~cm}$ between LDR

\section{and LED}

\begin{tabular}{|c|c|c|c|}
\hline \multirow{2}{*}{$\begin{array}{l}\text { Prescription of lens } \\
\text { (DIOPTERS) }\end{array}$} & \multicolumn{3}{|c|}{ LDR reading (K $\Omega$ ) } \\
\hline & $3 \mathrm{Cm}$ & $5 \mathrm{Cm}$ & $7 \mathrm{Cm}$ \\
\hline+15 & 7.21 & 6.74 & 6.42 \\
\hline+12 & 9.01 & 8.79 & 8.23 \\
\hline Reading glasses Add +2 & 10.3 & 10.4 & 10.1 \\
\hline-1.75 Cyl & 12.3 & 12.8 & 12.6 \\
\hline$-2.5-0.5-X 90$ & 13.09 & 13.8 & 13.5 \\
\hline$-2.5-0.5-X 180$ & 13.11 & 13.6 & 13.5 \\
\hline$-3 \mathrm{Sph}$ & 13.21 & 13.9 & 13.6 \\
\hline$-3.5 \mathrm{Sph}$ & 13.48 & 14.09 & 13.9 \\
\hline
\end{tabular}

resistance developed by the LDR. In the case of the lens having minus (-) power, it reduces the light intensity falling on the LDR, which makes LDR to increase its resistance.

From the above experimental study, it is observed that the high powered positive or negative optical lens will bring noticeable change in the light at focal point compared to the light falling on the lens. It is also to be noted and observed that the back vertex distance from the lens to LDR also plays a vital role. The light intensity experiencing by a subject varies as the change in the distance between the back vertexes of optical lens to an eye. This is proved in Table 2.

\section{Changes in the Human Physiological Variables when Subject Exposure to Light Though Optical Glasses}

In view of the fact that, optical powered glasses influences and

$$
E=I / d^{2}
$$

alters the light intensity experienced by the eye. Here, different illumination levels can cause a fusion of variations in many physiological parameters like alterations in subjective alertness, incidence of slow eye movements; plasma melatonin hormone, core body temperature and other ocular physiological changes. ${ }^{4-11}$ The lighting or the illumination on specific area caused by the luminous object is entirely dependent on the distance between illuminating area and the luminous object. The equation for illumination

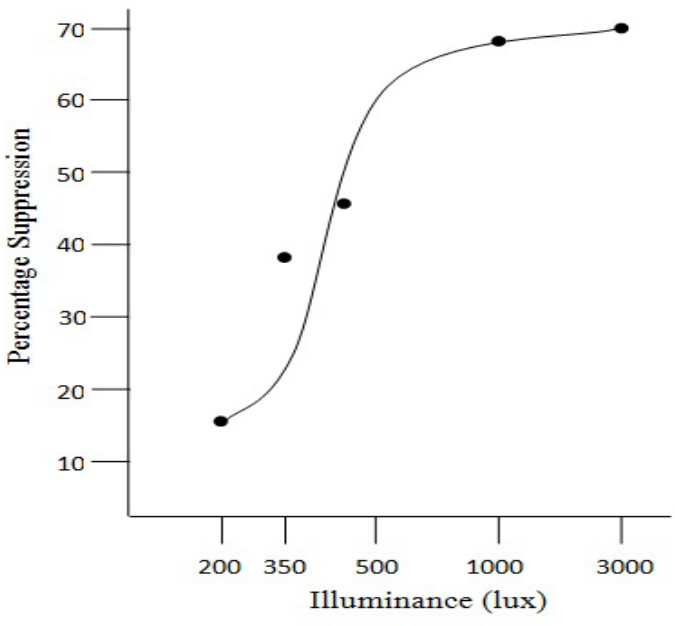

Figure 2: Response of a Melatonin Hormone to Different Illumination Levels. ${ }^{12}$

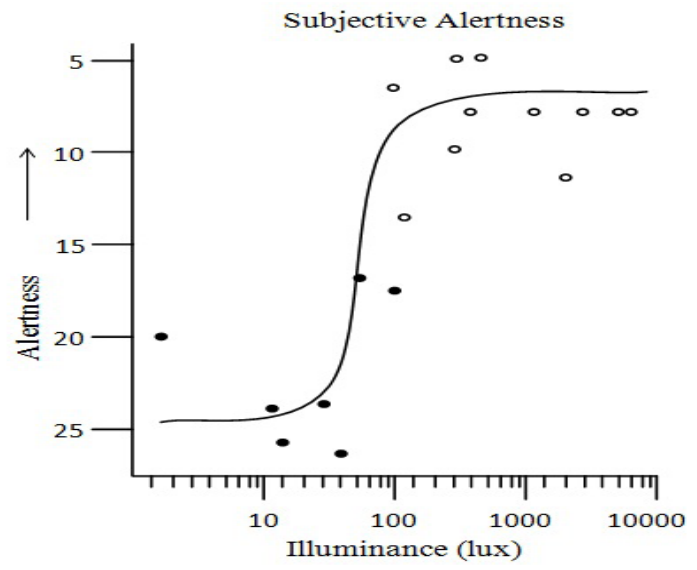

Figure 3: The subjective alertness response to with increment of illumination level. ${ }^{5}$

caused by the luminous object in a distance $\mathrm{d}$ is given in (Equation $1)$.

Where $E$ is the lighting caused by the luminous object at a distance $d, I$ is the amount of luminous intensity of a respective light source. Here, to comprehend the effect of light on human physiology, the release of amount of melatonin hormone in the subject is directly correlated with the amount of light intensity experiencing by the subject. It is a natural hormone which is initiated in pineal grand of the human brain. The synthesis of melatonin hormone is inhabited when the eye experiences the amount of light. As the intensity of the light increases, the release of melatonin hormone suppressed which results in increase of subjective alertness. Iain M. McIntyre, et al. (1989) enlightens the relation between the intensity of the light and the percentage suppression in melatonin hormone. This can be shown in Figure 2.

There are other physiological variables which are getting influenced by the bright illumination conditions with reference to variation in release of melatonin hormone. Subjective alertness is an additional physiological variable that is proportional to the light intensity experiencing by the subject and inversely proportional to release 
of melatonin hormone. Christian Cajochen (2007) correlated various physiological parameters with the light intensity experiencing by the subject with respect to melatonin hormone. More than $50 \%$ of release of melatonin hormone is suppressed when the subject is exposed to the illumination beyond 100 Lux. Then, the subjective alertness is increased when subject is exposed to illumination beyond 100 lux. Incidence of Slow eye movements is another human physiological change which is correlated with the release of melatonin hormone. The incidence of slow eye movements is getting increased with increase in the release of melatonin hormone. Release of melatonin hormone, subjective alertness and incidence of slow eye movements are inter-correlated physiological parameters with change in illumination conditions experiencing by the subject. The response of these physiological parameters towards different illumination rate with respect to melatonin hormone is shown below in Figure 3 and Figure 4. Here, closed symbols are to be identified as an individual, where suppression. The variation in these physiological changes due to different illumination conditions experiencing by the subject also brings numerous changes in the neurological behavior of the human system of melatonin hormone to less than $50 \%$, open symbol shows as an individual where melatonin hormone is suppressed beyond 50\% which can bring alterations various physiological and neurological parameters.

\section{ELECTROENCEPHALOGRAPHIC CHANGES WHEN TO EXPOSURE TO DIFFERENT ILLUMINATIONS}

\section{Effects on EEG frequencies}

Due to numerous changes in the human physiological variables when exposed to different illumination ranges, it also affects the rhythm of the neurological behavior. Here, Visual Cortex in occipital region is the responsible for the central vision of the human system. Central intent up on the alpha frequency in the occipital region shows vital association between neural changes and light intensity. When the subject eyes are closed or not experiencing light intensity visually, it is observed that the increment in alpha activity in the occipital region. Usually, alpha frequency is the state which occurs when the subject is in relaxing mode. Alpha blocking is another phenomenon which alters the alpha activity. This

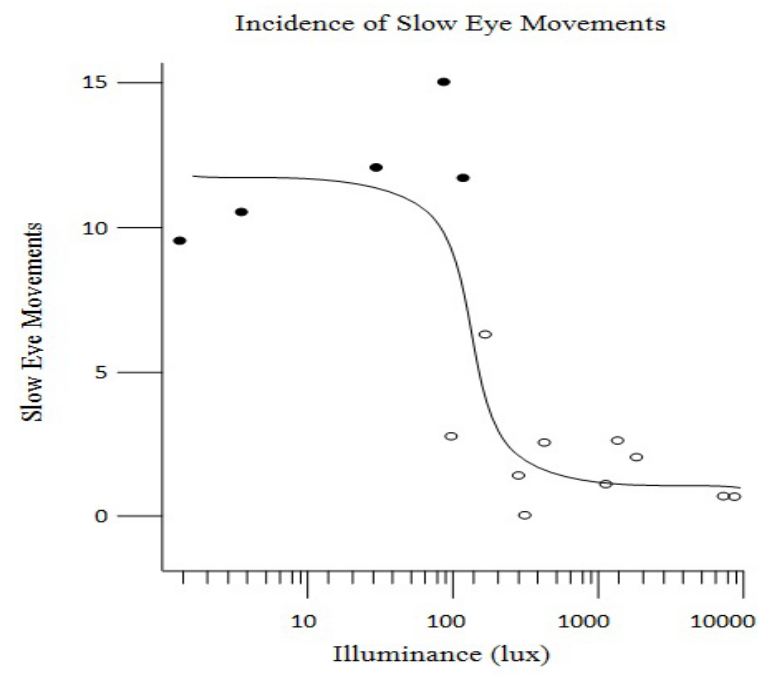

Figure 4: The reduction of incidence of slow eye movements with increase of illumination levels. ${ }^{5}$ occurs when the subject is exposed to light and allowed to do any mental activity. This makes the reduction in alpha activity and increase the frequency. Alpha rhythm replicates changes in physiological variables with increment in illumination levels. It can be illustrated that less illuminated environment or no light cause increase of alpha rhythm in the occipital region of the EEG. The frequency range of Alpha exhibits the properties of sleep spindles which occur between two states of alertness which ranges from 7-14 Hz. ${ }^{13}$

\section{Effect on EEG Power density}

The effect on EEG power density is the premier electroencephalographic changes due to exposure of subject to more illuminating conditions. Christian Cajochen, et al. (2000) gives the relation between the percentages of total power to the different range of the Illuminance shown in Figure 5. EEG power density of frequency in range of $5-9 \mathrm{~Hz}$ has been suppressed.

\section{NEURAL BEHAVIOR WHEN SUBJECT WITH IMPROPER FOCUSED LENSES}

Changes in Neural behavior are replicated in the readings of EEG when a subject is using improper focused lenses or with incorrect prescription which doesn't match to the refractive error are analyzed and verified in the laboratory using subjective and experimental analysis.

\section{Experimental Procedure}

This experimental study was carried out on 80 subjects, 38 male and 42 female participants of age ranges from 20-55 available in the university. In these participants, 52 subjects are with myopia and 28 subjects are with hypermetropia. All the subjects are corrected with different optical positive and negative powered glasses with different prescription given by the licensed ophthalmologist. All the participants were called individually to the laboratory which is temperature is maintained at around $25^{\circ} \mathrm{C}$. The prescription of every individual participant is noted. Now, $\mathrm{AgCl}$ electrodes are placed over the scalp in frontal and occipital regions of the international 10-20 standard. The reference electrode is placed with electrode gel on the left ear. ${ }^{14-17}$

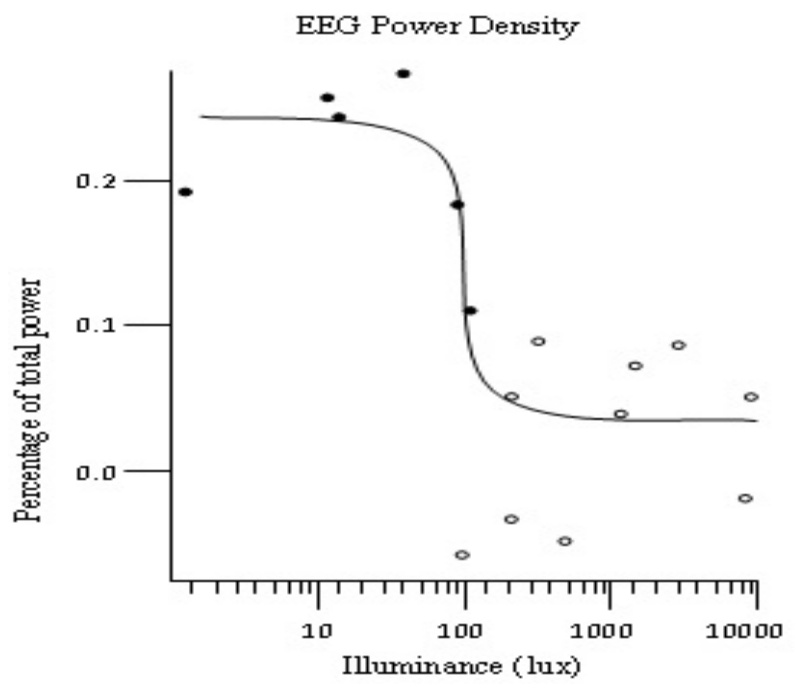

Figure 5: Percentage Reduction of EEG Power Density of frequency 5-9 $\mathrm{Hz}$ with Increment of Illumination. 


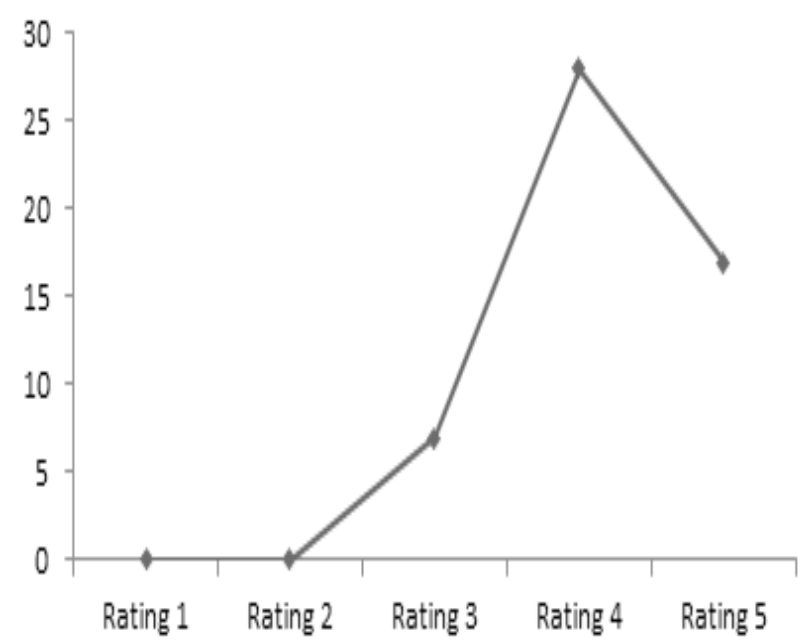

Figure 6: Subjective analysis performed on 52 subjects with Myopia.

Now each participant was asked to wear a different prescription powered optical glass than suggested by the ophthalmologist. Each participant was asked to read the chart with good font size at the distant or to read a book with an improper focused lens. The resultant changes in the EEG readings are analyzed.

\section{Observation}

As it is expected, when the subject tries to read the chart with improper focused lens, it is found that the subject is using more mental ability than usual. The characteristics of the frontal region frequency changes when the subject tries to focus and tries to read or approximate the chart placed in the laboratory. This makes increment in the frontal frequency. The frequency response for the mental activity done by the subject is showing up in frontal region as $12-25 \mathrm{~Hz}$. This can be considered as lower beta frequency. The state of occurrence of the beta frequency activity in frontal and front polar region is when subject applies more mental activity than usual.

\section{DISCUSSION}

After completion of experimental analysis, each individual subject was asked to give the rating for their mental stress used to read the chart with different prescription with improper focused optical powered glass suggested by the ophthalmologist. The ratings for this activity were asked to give by the subject from 1 to 5 . Rating 1 show as no mental stress ability used to read the chat or to focus an object. Where rating 5 shows as high mental stress activity is performed by the subject. Maximum of the subjects participated in the subjective analysis have given a rating of 4 to 5 . This shows improper focused optical powered glasses cause subject to use more mental activity than usual. It is found that similar characteristics between experimental and subjective analysis. The graphical representation of ratings given by subjects with myopia and hypermetropia to carry out the subjective analysis is given below. Optical powered lens is used to correct the difficulty of refractive errors in the ocular sensing. If the prescription given by the licensed ophthalmologist is not matching to the refractive error of the subject brings various noticeable consequences in the human neurological and physiological parameters. Initially, improper

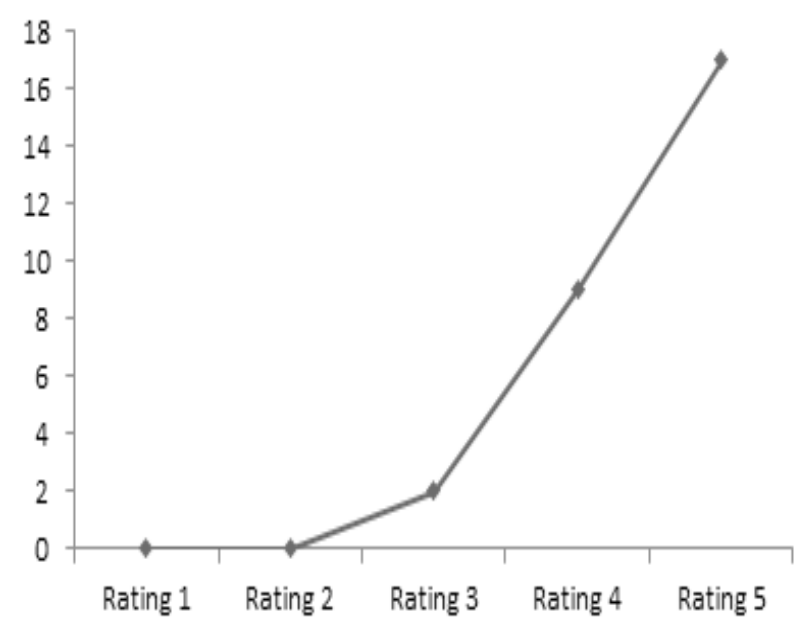

Figure 7: Subjective analysis performed on 28 subjects with Hypermetropia.

vision causes the subject to feel fatigue and strain. Long time stains to the eye while acquiring vision through improper focused lens results in headache. Squinting is the noticeable consequence and symptom when the subject is using different powered optical lens. It is recommended to the physician or EEG practitioner that to confirm optical powered glasses used by the subject is able to correct the refractive error of eye properly. If not, the frontal frequency gets increased and may suspect as seizures or other any abnormality.

\section{CONCLUSION}

Light through optical powered glasses can bring a numerous amendments to the physiological parameters of the human system. These changes in the physiological parameters lead to noticeable alterations in the neurological parameters. Hence, in this study, the grounds for the alterations in light intensity through the optical powered lens have been verified. Light dependent resistor test has been performed to prove and analyze alterations of light intensity through optical powered glasses. It is found that the positive powered glasses increases the light intensity through optical powered glasses. Because, it converges all the light rays at a focal point. This make subject to experience more light intensity than usual. While, negative powered optical glasses diverges all the light rays falling on the lens make the subject to feel less light intensity than normal.

It is also observed that improper and unfocused vision through optical lens make subject feel fatigue and which is prone to the headache and development of seizures in human brain. Experimental and subjective analyses are performed on 80 subjects to confirm that misfocused or unclear vision increases the mental activity when each subject is trying to focus and to have a clear vision. It is proved that increment in frontal frequency to the lower beta frequency range shows that subject applied more mental stress than usual. Subjective analysis has been performed individually and separately for the subjects using positive and negative powered glasses. It shows similar characteristics to the experimental analysis that subjects feel to apply more mental activity like problem solving. 


\section{CONFLICT OF INTEREST}

There is no conflict of interest

\section{ABBREVIATION}

EEG: Electroencephalogram; EOG: Electroculogram; LDR: Light Dependent Resistor.

\section{REFERENCES}

1. Medithe $\mathrm{J}$ and Nelakuditi U. "Study of normal and abnormal EEG", 2016 3rd International Conference on Advanced Computing and Communication Systems (ICACCS), 2016. https://doi.org/10.1109/icaccs.2016.7586341.

2. Krishnan RS. "Scattering of light in optical glasses", Proceedings of the Indian Academy of Sciences-Section A, 1936;3(3)211-20.

3. Medithe $\mathrm{J}$ and Nelakuditi $U$. "Removal of ocular artifacts in EEG", 2016 10th International Conference on Intelligent Systems and Control (ISCO), 2016. https://doi.org/10.1109/isco.2016.7726941.

4. Myers BL, Badia P. Immediate effects of different light intensities on body temperature and alertness. Physiology \& behavior. 1993;31;54(1):199-202.

5. Cajochen C. Alerting effects of light. Sleep medicine reviews. 2007;31;11(6):453-64.

6. J. Park, B. Min, Y. Jung, H. Pak, Y. Jeong and E. Kim, "Illumination influences working memory: An EEG study", Neuroscience. 2013;247;386-394. https:// doi.org/10.1016/j.neuroscience.2013.05.016; PMid:23694704

7. Cajochen C, Zeitzer JM, Czeisler CA, Dijk DJ. Dose-response relationship for light intensity and ocular and electroencephalographic correlates of human alertness. Behavioural brain research. 2000; 31;115(1):75-83.
8. Maher AM, Kirkup L, Swift P, Martin D, Searle A, Tran Y, Craig A. Effect of luminance level on electro-encephalogram alpha-wave synchronisation. Medical and Biological Engineering and Computing. 2001;1;39(6):672-7.

9. Toscani M, Marzi T, Righi S, Viggiano MP, Baldassi S. Alpha waves: a neural signature of visual suppression. Experimental brain research. 2010;1;207(34):213-9.

10. Cajochen C, KRÄUCHI K, Danilenko KV, WIRZ-JUSTICE AN. Evening administration of melatonin and bright light: interactions on the EEG during sleep and wakefulness. Journal of sleep research. 1998;1;7(3):145-57.

11. Brown BB. Recognition of aspects of consciousness through association with EEG alpha activity represented by a light signal. Psychophysiology. 1970;1;6(4):442-52.

12. Mclntyre IM, Norman TR, Burrows GD, Armstrong SM. Human melatonin suppression by light is intensity dependent. Journal of pineal research. 1989;1;6(2):149-56.

13. Narayan S, Subramanian S and Gaur G, "Low luminance/eyes closed and monochromatic stimulations reduce variability of flash visual evoked potential latency", Annals of Indian Academy of Neurology, 2013;16(4):614. https://doi. org/10.4103/0972-2327.120492; PMid:24339591 PMCid:PMC3841612.

14. R. Cooper, J. Osselton and J. Shaw, EEG technology, 1st ed. London: Butterworths, 1969.

15. E. Niedermeyer and F. Lopes da Silva, Electroencephalography, 1st ed. Philadelphia: Wolters Kluwer Health, 2011.

16. Medithe $\mathrm{J}$ and Nelakuditi $\mathrm{U}$. "Study on the Impact of Light on Human Physiology and Electroencephalogram", Journal of Biomimetics, Biomaterials and Biomedical Engineering, 2016;28:36-43. https://doi.org/10.4028/www. scientific.net/JBBBE.28.36.

17. Bong SZ, Murugappan M, Yaacob S. Methods and approaches on inferring human emotional stress changes through physiological signals: A review. International Journal of Medical Engineering and Informatics. 2013;1;5(2):152-62

\section{PICTORIAL ABSTRACT

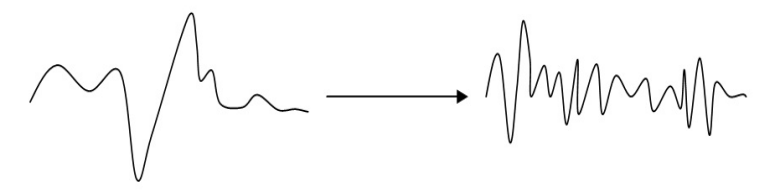 \\ Variation in EEG due to Light through Optical glasses}

\section{SUMMARY}

- The influence of light on EEG is given but it is correlated with Variation of light intensity through optical glasses

- Some changes in physiological parameters like release of melatonin hormone etc, which in turn bring changes brain electrical activity like suppression of alpha activity in occipital region

- Light through optical glass bring noticeable variation in the aptitude of the blink, contaminates in the frontal electrodes

- Light dependent resistor test has been performed to prove and analyze alterations of light intensity through optical powered glasses.

- Greater than equal to $\pm 3 \mathrm{D}$ bring noticeable change

- It is also observed that improper and unfocused vision through optical lens make subject feel fatigue and which is prone to the headache and development of seizures in human brain.

- Experimental and subjective analyses are performed on 80 subjects to confirm that misfocused or unclear vision increases the mental activity when each subject is trying to focus and to have a clear vision

- Brightness coating has to be made to negative powered glasses and dullness coating is to be applied to positive powered glasses to neutral the light variation in optical glasses

- It is recommended to physician that, Optical Glass test whether suits the refractive error are to be checked before the test 


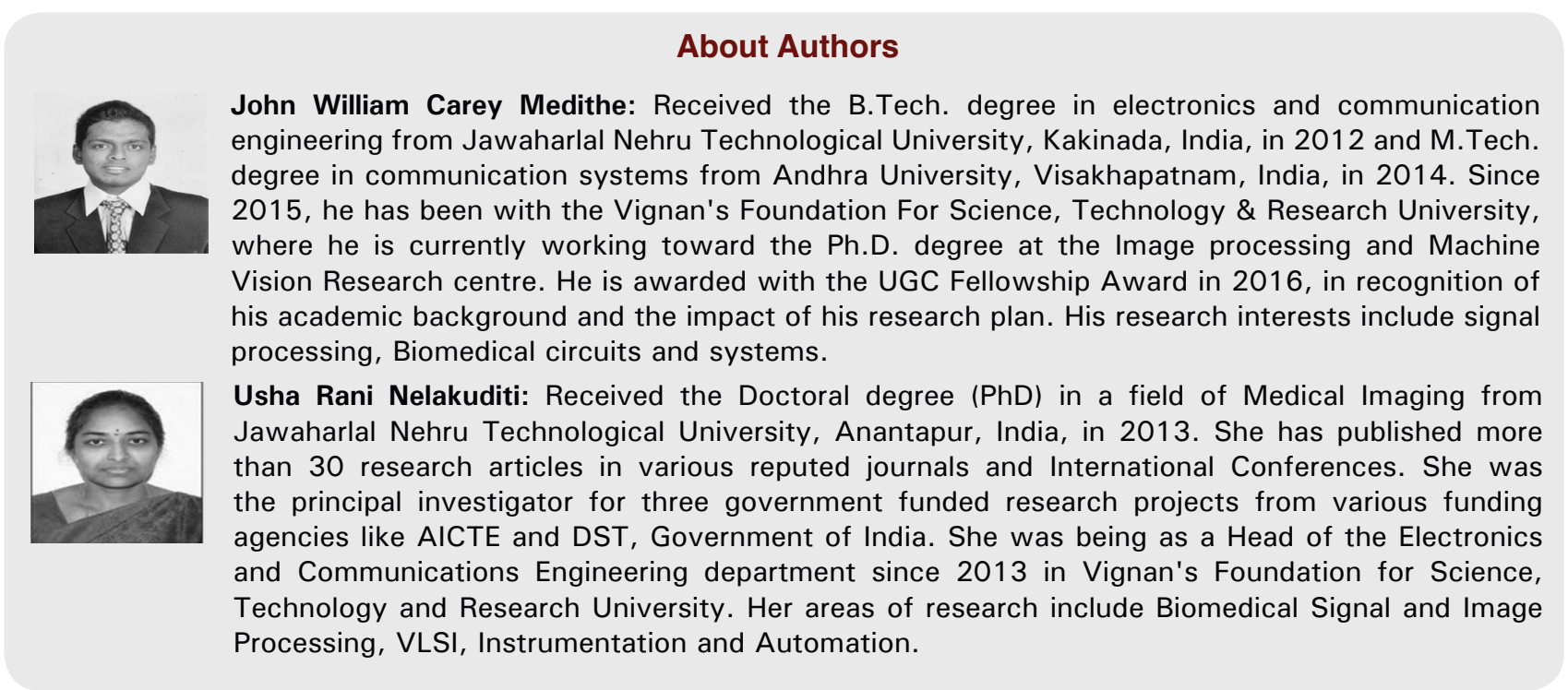

Cite this article: Medithe JWC, Nelakuditi UR. Influence of light Through Optical Glasses on Electroencephalogram. Indian J of Pharmaceutical Education and Research. 2017;51(2s):S54-S60. 